\title{
Complex organic molecules in diffuse clouds along the line of sight to Sagittarius B2 (Corrigendum)
}

\author{
V. Thiel ${ }^{1}$, A. Belloche ${ }^{1}$, K. M. Menten ${ }^{1}$, R. T. Garrod ${ }^{2}$, and H. S. P. Müller ${ }^{3}$ \\ ${ }^{1}$ Max-Planck-Institut für Radioastronomie, Auf dem Hügel 69, 53121 Bonn, Germany \\ e-mail: vthiel@mpifr-bonn.mpg.de \\ 2 Departments of Chemistry and Astronomy, University of Virginia, Charlottesville, VA 22904, USA \\ ${ }^{3}$ I. Physikalisches Institut, Universität zu Köln, Zülpicher Str. 77, 50937 Köln, Germany \\ A\&A, 605, L6 (2017), DOI: 10.1051/0004-6361/201731495
}

Key words. ISM: molecules - radio lines: ISM - astrochemistry - ISM: individual objects: Sagittarius B2(N) - errata, addenda

Corby (2016) reported the detection of several complex organic molecules in absorption at various velocities toward Sgr B2 based on the PRIMOS survey ${ }^{1}$. In particular, she detected $\mathrm{HC}_{3} \mathrm{~N}$, $\mathrm{CH}_{3} \mathrm{CHO}$, and $\mathrm{NH}_{2} \mathrm{CHO}$ in the Scutum arm at a systemic velocity of about $25 \mathrm{~km} \mathrm{~s}^{-1}$, but $\mathrm{CH}_{3} \mathrm{OH}$ was not detected in absorption at this velocity due to contamination with maser emission. Therefore, the detections of $\mathrm{CH}_{3} \mathrm{OH}$ and $\mathrm{CH}_{3} \mathrm{CN}$ in the Scutum arm reported in Thiel et al. (2017) remain new, and they confirm the presence of complex organic molecules in spiral arm clouds outside the galactic center first reported by
Corby (2016). We became aware of the PRIMOS results of Corby (2016) on complex organic molecules only after the publication of Thiel et al. (2017). The results and conclusions of our Letter are not affected.

\section{References}

Corby, J. 2016, PhD Thesis, University of Virginia

Thiel, V., Belloche, A., Menten, K. M., Garrod, R. T., \& Müller, H. S. P. 2017, A\&A, 605, L6 